\title{
Chronic fatigue syndrome: a follow up study
}

\author{
D Bonner, M Ron, T Chalder, S Butler, S Wessely
}

\begin{abstract}
Forty-six of 47 patients diagnosed as having chronic fatigue and offered treatment four years previously were followed up. Twenty-nine patients were interviewed, three patients refused an interview, and information on the remaining 14 was obtained from their general practitioners. All the instruments used at interview had been used in the initial study. The long-term prognosis for patients with chronic fatigue syndrome who have initially responded to treatment is good. Spontaneous recovery in those who declined or who did not benefit from treatment is unlikely. Patients who continue to fulfil the criteria for chronic fatigue syndrome four years after they were initially diagnosed are likely to have had more somatic disorders, to have been more fatigued, and to have had a previous psychiatric history when they were initially assessed.
\end{abstract}

$(\mathcal{F}$ Neurol Neurosurg Psychiatry 1994;57:617-621)

Over the past decade much attention has been focused on the syndrome variously described as myalgic encephalomyelitis, postviral fatigue syndrome, and latterly chronic fatigue syndrome. This plethora of names and the frequency of descriptions is testament to the confusion surrounding the syndrome. The term chronic fatigue syndrome has been proposed to describe disabling fatigue of at least six months' duration of uncertain aetiology. ${ }^{1-3}$ The cause of the syndrome remains to be determined, but there is a complex interaction between biological and psychological substrates.

A series of studies has shown that between 50 and $80 \%$ of subjects with chronic fatigue syndrome seen in specialist centres or primary care settings fulfil criteria for psychiatric disorders. ${ }^{4-9}$ Although reports of chronic fatigue can be found as far back as 1869 with Beard's description of neurasthenia or nervous exhaustion, ${ }^{10}$ systematic studies of the disorder have only been undertaken in the past few years and therefore information about the natural history and the effects of treatment is limited. Anecdotal reports of a large series suggested an extremely gloomy natural history. ${ }^{11}$ A follow up study of subjects seen at an infectious disease clinic was only slightly more optimistic, ${ }^{12}$ as most patients remained symptomatic two to four years after assessment. Treatment was not offered in either of these studies.

In this study we followed up a group of patients seen at the National Hospital for Neurology and Neurosurgery four years previously who had been diagnosed as having chronic fatigue. ${ }^{6}$ These patients were initially assessed before the current criteria for chronic fatigue syndrome became available, but most would have satisfied the criteria retrospectively; only two patients were excluded because of a life-long history of fatigue (that is, no definite onset).

These 47 patients were initially offered treatment in an open, uncontrolled trial using cognitive behaviour therapy. Antidepressants were offered to those patients who fulfilled diagnostic criteria for depression. ${ }^{13}$ Treatment followed cognitive behavioural principles derived from work on the management of chronic pain but adapted for the particular problems of chronic fatigue syndrome. ${ }^{14} 15$

We decided to follow up this group of patients to determine if treatment gains were maintained, what happened to those who refused treatment, and to elucidate further the role of psychiatric morbidity in the prognosis of chronic fatigue syndrome.

\section{Subjects and methods \\ SUBJECTS}

Forty-six of the 47 subjects who took part in the original study were contacted by post four years later. The case notes of the remaining patient could not be traced. Those who did not respond to the initial letter were sent a second. Thirty-two (70\%) patients responded (18 women, 14 men).

Of those who responded, $29(91 \%)$ agreed to be interviewed. Nine $(28 \%)$ were seen at home, 11 (34\%) at the National Hospital for Neurology and Neurosurgery, and nine were interviewed by telephone. The general practitioners of the 14 patients who did not respond were contacted by telephone to obtain information about their physical and mental health during the follow up period.

\section{INSTRUMENTS}

Three questionnaires were sent by post when the patients were initially contacted. One was a fatigue questionnaire modified from that devised for the previous study. ${ }^{616}$ Scores ranged from 0 to 10 ; the higher the score the greater the fatigue. The results of the initial fatigue questionnaires were corrected to make the index scores and follow up scores directly 
comparable. The other two questionnaires were the General Health Questionnaire (GHQ-12) ${ }^{17}$ and the Hospital Anxiety and Depression (HAD) Questionnaire. ${ }^{18}$

At follow up patients were classified as still having chronic fatigue syndrome if they fulfilled the following criteria: (a) scoring above 4 on the fatigue questionnaire; (b) being fatigued for six months or more; and (c) feeling fatigued more than $50 \%$ of the time. These criteria are based on guidelines set out by Sharpe $e t a l^{2}$ in their recommendations for a working case definition of chronic fatigue syndrome.

At the interview the following instruments were used: (a) the schedule for affective disorder and schizophrenia (SADS). ${ }^{19}$ This is a standardised psychiatric interview developed to provide current and lifetime research diagnostic criteria (RDC) diagnoses. (b) The Beck depression inventory (BDI). ${ }^{20}$ (c) The modified somatic discomforts questionnaire. ${ }^{21}$ This consists of a list of 17 common symptoms which are rated on a three-point scale (1-3) indicating whether they were a slight problem (1), a moderate problem (2), or a severe problem (3). (d) A self-assessment scale of functional impairment. ${ }^{22}$ This records functional disability and consists of four visual analogue scales covering ability to work, home management, and social and private leisure activities. Patients were asked how much their problems affected their functioning in each of these designated areas. The scores of the four visual analogue ratings of functional impairment were summed and treated as a single variable, labelled 'impairment index' (range 0-8). All these measures had been used in the previous study. ${ }^{13}$ (c) Self-rated global improvement. Subjects were also asked to rate their overall improvement since the initial assessment on a percentage scale, taking $0 \%$ as the point they felt most fatigued and with $100 \%$ implying complete recovery.

\section{STATISTICS}

Statistical procedures were performed using the Statistical Package for Social Scientists (SPSS). ${ }^{23}$ Non-parametric statistics used were the Mann-Whitney U test and the $\chi^{2}$ test. All significance testing was two-tailed.

\section{Results}

There was no significant difference between the respondents and non-respondents in age, sex, age at onset of fatigue, duration of fatigue, severity of depression, or the presence of a previous psychiatric history at index assessment.

The mean age at follow up of the 29 patients who were interviewed was 41 years (range 23-64). Their mean age at the onset of fatigue was 33.8 years (range 17-57.5) and they had had the symptom of fatigue for a mean of 3.2 years (range $0.5-10$ ) when they were initially assessed.

Twenty two $(76 \%)$ of these patients had accepted treatment when first seen and of these 17 had successfully completed it. Three patients discontinued treatment and two patients felt no benefit at the completion of treatment. Seven of the patients seen at follow up had refused treatment when initially assessed.

\section{SELF-RATED GLOBAL IMPROVEMENT}

The 29 subjects were divided into two groups: group 1-those who felt no or only a slight improvement $(<50 \%$ score on the selfrated global improvement scale) (13 patients); and group II-those who felt they had recovered or almost recovered $(>50 \%$ score on the self-rated global improvement scale) (16 patients). Table 1 summarises these findings.

Of the 13 subjects who reported little or no improvement, 11 fulfilled RDC criteria for a current psychiatric diagnosis of depression and had the same diagnosis when assessed for the initial study. Their reported level of functional impairment at follow up (mean 20.2 , range 8-30) was not significantly changed from the initial assessment (mean 20.57, range 13-31). Subjects were impaired in all the areas assessed. Eight patients in this group had been unable to return to work since becoming fatigued and two had been housebound since the onset of their fatigue symptoms six and seven years previously. Eight subjects currently fulfilled the criteria for chronic fatigue syndrome.

Of the 16 subjects who felt they had recovered or almost recovered only one fulfilled the criteria for a psychiatric diagnosis. This patient was diagnosed as having postpartum depression after childbirth six months previously. The functional impairment of subjects in this group was minimal at follow up (mean $2 \cdot 78$, range $0-11$ ). At the initial assessment there was no significant difference in functional impairment between the two groups. Two patients in this group continued to fulfil the criteria for chronic fatigue.

CHRONIC FATIGUE SYNDROME

Ten $(34 \%)$ of 29 patients interviewed continued to fulfil the criteria for chronic fatigue syndrome at the four year follow up. Of these, seven also had an RDC psychiatric diagnosis (major depression in five and minor depression in two) and this contrasted with only five of the 19 without chronic fatigue syndrome. The diagnoses in this group were

Table 1 Self-rated global improvement. Values given are numbers of patients or questionaire score

\begin{tabular}{lll}
\hline & $\begin{array}{l}\text { No or little } \\
\text { improvement } \\
(<50 \%) \\
(n=13)\end{array}$ & $\begin{array}{l}\text { Improved/ } \\
\text { recovered } \\
(>50 \%) \\
(n=16)\end{array}$ \\
\hline $\begin{array}{l}\text { Psychiatric disorder at } \\
\text { follow up }\end{array}$ & $11(11)$ & $1(13)$ \\
$\quad$ (at index interview) & 8 & 2 \\
$\begin{array}{l}\text { CFS at follow up } \\
\text { Completed treatment }\end{array}$ & 6 & 14 \\
$\begin{array}{l}\text { Functional impairment } \\
\text { score at follow upt }\end{array}$ & $20 \cdot 2(8-30)$ & $2 \cdot 78(0-11)$ \\
\hline
\end{tabular}

${ }^{\star} \mathrm{CFS}=$ Chronic fatigue syndrome. †Mean (range). 
somatisation disorder (two patients), major depression (one patient), minor depression (one patient) and anxiety disorder (one patient). The difference between the groups is statistically significant $(p<0.05)$. Table 2 reports other differences between the two groups. Thus patients in the group with persisting chronic fatigue syndrome had a greater functional impairment, experienced greater somatic discomfort and had higher scores for anxiety and depression.

The ratings obtained during the initial assessment by those who fulfilled the criteria for chronic fatigue syndrome at follow up were compared with those of the rest of the group to ascertain possible differences of prognostic value (see table 3 ).

There was no difference in age of onset, sex, or duration of fatigue at index assessment between the two groups, nor was there any difference in the severity of depression as assessed by their BDI and HAD scores. Those who continue to have chronic fatigue syndrome at follow up, however, saw themselves as experiencing greater somatic discomfort and had significantly greater fatigue scores at the index assessment.

A change in the nature of the symptoms appeared to have taken place during the follow up period. Only five of the 10 patients who continue to fulfil the criteria for chronic fatigue syndrome felt that their current disorder was fatigue. Two patients felt that it was low mood, two reported poor concentration, and one patient felt it was recurrent chest pain due to angina. In contrast, at the initial

Table 2 Physical and psychiatric morbidity in patients with chronic fatigue syndrome: follow up rating

\begin{tabular}{|c|c|c|}
\hline & \multicolumn{2}{|c|}{ Mean ( $95 \%$ confidence interval) score at follow up } \\
\hline & $\begin{array}{l}\text { CFS at follow up } \\
(n=10)^{\star}\end{array}$ & $\begin{array}{l}\text { No CFS at follow up } \\
(n=19) t\end{array}$ \\
\hline $\begin{array}{l}\text { Impairment index } \\
\text { GHQ } \\
\text { BDI } \\
\text { HAD }\end{array}$ & $\begin{array}{r}21.5(16.9 \text { to } 26 \cdot 1) \\
5 \cdot 7(3.6 \text { to } 7 \cdot 7) \\
8 \cdot 7(5 \cdot 0 \text { to } 12 \cdot 4) \\
18 \cdot 9(13.6 \text { to } 24 \cdot 2)\end{array}$ & $\begin{array}{l}5.9(2.5 \text { to } 9.3 \ddagger \\
1.8(0.5 \text { to } 3.1) \ddagger \\
3.0(0.5 \text { to } 5.0) \ddagger \\
7.7(4.3 \text { to } 11.1) \ddagger\end{array}$ \\
\hline $\begin{array}{l}\text { Somatic discomfort } \\
\text { questionnaire }\end{array}$ & $19.5(13.9$ to $25 \cdot 1)$ & $6.7(3.5$ to 9.9$) \ddagger$ \\
\hline
\end{tabular}

Abbreviations: CFS $=$ Chronic fatigue syndrome; GHQ $=$ General Health Questionnaire; BDI $=$ Beck Depression Inventory; and $\mathrm{HAD}=$ Hospital Anxiety and Depression scale. * Seven patients fulfilled the RDC diagnosis at follow up.

+ Five patients fulfilled the RDC diagnosis at follow up; $p<0.05\left(\chi^{2}\right.$ test $)$

$\neq \mathrm{p}<0.01$; Mann-Whitney U test.

Initial ratings and outcome of fatigue

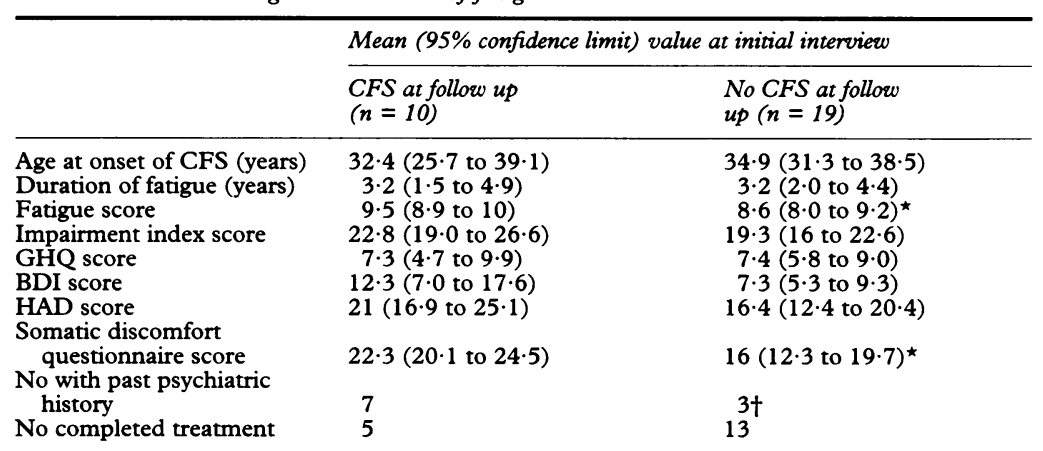

Abbreviations: CFS = Chronic fatigue syndrome; GHQ = General Health Questionnaire; BDI $=$ Beck Depression Inventory; and HAD = Hospital Anxiety and Depression scale.

$\ddagger \mathrm{p}<0.01$; Mann-Whitney U test.

${ }_{\mathrm{t}}^{\mathrm{p}}<0.05$; Mann Whitney $U$ test. $\dagger_{\mathrm{p}}<0.01 ; \chi^{2}$ test. assessment fatigue was the most prominent disorder in all of these subjects.

\section{TREATMENT OUTCOME}

Of the 22 patients who accepted treatment 17 were reported to be improved at the completion of treatment. After four years this improvement was sustained in 14 patients as determined by their self-rated global improvement scores. Two of the three patients who relapsed have had recent admissions to a psychiatric ward for depression. All three are currently attending psychiatric outpatient clinics and are receiving antidepressants. Of the five patients who did not successfully complete treatment none had spontaneously improved at the four year follow up. Seven of the patients seen at follow up had refused treatment; of these two had made a spontaneous recovery.

\section{ILLNESS ATTRIBUTION}

All subjects interviewed at follow up were asked about their illness attribution-whether they believed that the cause of their illness was physical, mainly physical, or whether it was psychological or mainly psychological. Nine of the 10 patients with chronic fatigue syndrome attributed their illness to a physical cause, but so did 12 of the 19 patients without chronic fatigue syndrome. This difference did not reach statistical significance.

\section{PHYSICAL AND PSYCHIATRIC MORBIDITY IN}

\section{NON-RESPONDENTS}

The general practitioners of all the 14 nonrespondents were contacted. Six (43\%) subjects were reported to be well physically and mentally when last seen by their general practitioner. All of these had successfully completed treatment. Four patients were reported to be still unwell and visiting their general practitioners every three to four months with fatigue as their main disorder. Two of them were wheelchair bound when first seen and remained so. All of these had refused treatment when initially seen. One patient had an $\mathrm{RDC}$ diagnosis of major depression and three had a diagnosis of somatisation disorder when initially assessed. One patient had recently been seen at the National Hospital for Neurology and Neurosurgery for a disability allowance appraisal. Her original diagnosis of somatisation disorder had been changed to one of hysterical conversion disorder. In four cases the general practitioners had not seen the patients for several years and could not give information. In one of these the general practitioner had asked for the patient to be removed from his practice because of noncompliance with treatment. The patient was still confined to a wheelchair when the general practitioner last saw him two years previously.

When the general practitioner reports of the patients' current status were taken as evidence of the presence/absence of fatigue there was a significant association between completion of treatment and the absence of chronic fatigue syndrome at follow up. Thus from 
patient and general practitioner reports at four years, $20(87 \%)$ of the 23 patients who successfully completed treatment remained well, whereas only two (13\%) of the 16 who refused or did not complete treatment had made a spontaneous recovery.

\section{Discussion}

We acknowledge several shortcomings in this study. The number of subjects seen at follow up was smaller than in the original study and in the initial study the treatment group was uncontrolled and therefore non-blind and non-randomised. Nevertheless it is hoped that the findings presented here will shed some light on the long-term prognosis of chronic fatigue syndrome and the long-term effects of treatment of a kind which is now commonly used in these patients.

Patients were reassessed four years after they had been seen at the National Hospital for Neurology and Neurosurgery and diagnosed as having chronic fatigue. In the initial follow up ${ }^{13} 70 \%$ of the group who accepted treatment described themselves as 'better' or 'much better' when treatment was completed and at three months this had been sustained. Follow up at four years continues to show that treatment benefits are being maintained. There was a trend for the initial treatment response to be associated with the continued absence of chronic fatigue syndrome at four years. This reached conventional statistical significance when general practitioner assessments of current functioning were included. We therefore conclude that there is a strong association between successful completion of treatment and the absence of functional disability at the four year follow up.

Those patients who continued to be impaired at follow up had marked functional disabilities resulting in long-term unemployment and continuing costs to their health services. The issue of disability payments for patients with chronic fatigue syndrome is currently receiving attention and is the subject of controversy. Our results highlight the longterm benefits that may result from treatment. As treatment in the original study was nonrandom and all patients were offered cognitive behaviour therapy, with only those who were considered to be depressed offered antidepressants, we are unable to discuss the relative merits of cognitive behaviour therapy versus treatment with antidepressants. A randomised controlled trial of cognitive behaviour therapy by two of the authors (TC and SW) is nearing completion, however, and it is hoped that more information on this subject will soon be available.

One of the salient findings of our study is that at four years just over one third of our sample continues to fulfill the criteria for chronic fatigue syndrome. This is similar to the findings of Sharpe et al. ${ }^{24}$ In their study these workers followed up a group of 144 patients seen in an infectious disease clinic with a major disorder of fatigue of at least six weeks' duration. No treatment was offered.
They found that a third of patients remained impaired at two to four years compared with $73 \%$ followed up at six weeks to six months after the initial assessment. Although our treated outcome is similar to that of Sharpe et al, it should be emphasised that the National Hospital for Neurology and Neurosurgery is a tertiary referral centre and more severely affected patients who have not responded to other methods of treatment are likely to be referred. Also patients in the study of Wessely et $a l^{6}$ reported fatigue for a substantially longer period of time (more than six months compared with more than six weeks). Other follow up studies have been conducted over shorter periods and indicate greater morbidity in the short term. Thus Petersen and Schenck ${ }^{25}$ did not observe complete recovery in any of their series of 62 patients over one year and Valdini $e t a^{26}$ in a primary care setting reported an improvement in only a quarter of their sample at one year. Neither of these studies offered treatment. Although it is clear that the proportion of patients impaired does decrease with time, we have shown that treatment produced the improvement early and this improvement was maintained over time.

The prognosis of severe chronic fatigue syndrome appears to be associated with psychiatric morbidity and in particular depression. Eight of the nine patients in our series who had a current RDC diagnosis of depression fulfilled the criteria for current chronic fatigue syndrome; on the other hand, the three patients who had RDC diagnoses other than depression did not have chronic fatigue syndrome.

In descriptive terms many of the symptoms that are accompanied by chronic fatigue syndrome are similar to those of depression. It has been suggested that severe chronic fatigue syndrome and severe depression are largely indistinguishable in their symptom profiles. ${ }^{27}$ Several reviews have discussed these dilem$\operatorname{mas}^{828}$ and it remains difficult to disentangle the "cause and effect" conundrum associating the two. It has been postulated that in some patients persistent fatigue may be initiated by an acute viral infection with its usual symptoms of transient lassitude and malaise, which are then perpetuated in the psychologically vulnerable by relevant psychological factors. ${ }^{29}$ Another possibility is that patients who fulfill the criteria for chronic fatigue syndrome have a psychiatric illness which predominantly manifests as fatigue. In favour of this is the fact that many depressed patients often report physical symptoms similar to those of chronic fatigue syndrome. The presence of a psychiatric disorder before the presentation of fatigue would be consistent with this.

The presence of a psychiatric diagnosis before and after the development of fatigue suggests that for some patients fatigue is an integral part of depression. This association, however, is by no means clear cut, as not all patients with chronic fatigue syndrome have a psychiatric diagnosis, nor is the presence of a previous psychiatric diagnosis uniformly pre- 
sent in patients presenting with fatigue. Many diseases are aetiologically complex and chronic fatigue syndrome would not be unique in this respect. It is probable that it is not a disorder with a single specific cause but the outcome of interacting processes at biological and psychological levels. This would favour treatment approaches aimed at the psychiatric disturbance as well as dealing with the specific behavioural aspects of fatigue.

Attribution of illness to a physical cause does not appear to be as important a prognostic factor in the long term. Butler et al ${ }^{13}$ found that a poor response to treatment was significantly associated with attribution to a physical cause. Although there was a trend for this to be so at the four year follow up this finding was not statistically significant, and those who had a good response to treatment did not change their attribution over the years. In other words patients still believed they had had post-viral fatigue syndrome/ chronic fatigue syndrome/myalgic encephalomyelitis even after their recovery. In contrast with Sharpe et $a l^{24}$ we found no association between membership of the myaglic encephalomyelitis association and continuing morbidity at follow up. In our series only three of those interviewed were active members of the myalgic encephalomyelitis association at four year follow up.

We also found in this series that there was no evidence for neurological or physical illness developing over four years. New diagnoses were entirely psychiatric; however, some patients developed new somatoform disorders, one patient now being labelled as having conversion hysteria, another unexplained chest pain. Patients with multiple unexplained somatic symptoms are known to change attributed labels over time. It has been noted how patients with chronic disorders have switched to the label of myalgic encephalomyelitis over time, ${ }^{30}$ whereas Potts $S$ and Bass $C$ (unpublished data), in a longterm follow up study of patients with severe chest pain and normal coronary arteries, have noted how some have now acquired the label of myalgic encephalomyelitis. In our study we noted a two-way traffic. A further area of concern was that only half of those who fulfilled the diagnostic criteria for depression were receiving any treatment at follow up. Indeed, perhaps the most disturbing aspect of this study is that the morbidity of those who refused treatment appears to be high. This may indicate that the presence of severe somatic symptoms deflects doctors' attention and many patients who fulfill diagnostic criteria for treatment go untreated. Future studies or treatment should concentrate on this group who otherwise appear to have a bad prognosis.

Dr MA Ron is partly supported by the SCARFE Trust.

1 Holmes G, Kaplan J, Gantz N, et al. Chronic fatigue syndrome: a working case definition. Ann Intern Med 1988;319:1726-8.

2 Sharpe MC, Archard LC, Banatvala JE, et al. A reportchronic fatigue syndrome: guidelines for research. $f R$ Soc Med 1991;84:118-21.

3 Schluederberg A, Straus SE, Peterson P, et al. Chronic fatigue syndrome research: definition and medical outcome assessment. Ann Intern Med 1992;117:325-31.

4 Taerk GS, Toner B, Salit IE, Garfinkel PE, Ozersky S. Depression in patients with neuromyasthenia (benign myalgic encephalomyelitis). Int $\mathcal{f}$ Psychiatry Med 1987;17:49-56.

5 Manu P, Lane T, Matthews D. The frequency of chronic fatigue syndrome in patients with symptoms of persistent fatigue. Ann Intern Med 1988;109:554-6.

6 Wessely S, Powell R. Fatigue syndrome: a comparison of postviral fatigue with neuromuscular and affective disorpostviral fatigue with neuromuscular and affective
der. $\Im$ Neurol Neurosurg Psychiatry 1989;52:940-8.

7 Abbey S, Garfinkel P. Chronic fatigue and depression: cause, effect or covariate. Rev Infect Dis 1991;1(suppl 13):73-83.

8 David AS. Postviral fatigue syndrome and psychiatry. $\mathrm{Br}$ Med Bull 1991;47:966-88.

9 McDonald E, David A, Pelosi A, Mann A. Chronic fatigue in general practice attenders. Psychol Med. In press.

10 Wessely $\mathrm{S}$. The natural history of chronic fatigue and myalgia syndromes. In: Sartorius $\mathrm{N}$, Goldberg $\mathrm{D}$, Wittchennh, DP, et al. Psychological disorders in general medical settings. Bern: Hans Huber, 1990:82-97.

11 Behan $P$, Behan W. The postviral fatigue syndrome. CRC Crit Rev Neurobiol 1988;42:157-78.

12 Hinds G, McCluskey D. A retrospective study of the chronic fatigue syndrome. Proc $R$ Coll Physicians Edin chronic fatigue

13 Butler S, Chalder T, Ron M, Wessely S. Cognitive behaviour therapy in chronic fatigue syndrome. $\mathcal{F}$ Neurol Neurosurg Psychiatry 1991;54:153-8.

14 Pither C. Treatment of persistent pain. BMF 1989;299. 1239-40.

15 Sensky T. Patients' reaction to illness. BMF 1990;300: 622-3.

16 Chalder T, Berelowitz G, Pawlikowska T, et al. Development of a fatigue scale. $\mathcal{F}$ Psychosom Res 1993;37: 147-53.

17 Goldberg $\mathrm{D}$. The detection of a psychiatric illness by questionnaire. London: Oxford University Press, 1972.

18 Zigmund A, Snaith R. The hospital anxiety and depression scale. Acta Psychiatr Scand 1983;67:361-70.

19 Spitzer R, Endicott J. Schedule for affective disorders and spitzer R, Endicott J. Schedule for affective disorders and
schizophrenia. New York: New York State Psychiatric schizophrenia. N
Institute, 1978.

20 Beck A, Ward C, Mendelson M, Mock J, Erbaugh J. An inventory for measuring depression. Arch Gen Psychiatry 1961;4:561-71.

21 Wittenborn J, Buhler R. Somatic discomforts among depressed women. Arch Gen Psychiatry 1979;36:465-71.

22 Marks I. Behavioural psychotherapy: Maudsley pocket book of clinical management. Bristol: Wright, 1986.

23 Marija J. SPSS Base Manual. Chicago, Illinois: Norusis/SPSS Inc., 1988.

24 Sharpe M, Hawton K, Seagrott V, Pasvol G. Fatigue: follow up of referrals to an infectious disease clinic. $B M \mathcal{Y}$ 1992;305:147-52.

25 Petersen $P$, Schenck $C$. Chronic fatigue syndrome in Minnesota. Minn Med 1991;74:21-6.

26 Valdini A, Steinhardt S, Valicenti J, Jaffe A. A one year follow up of fatigued patients. $\mathcal{F}$ Fam Pract 1988;26: 33-8.

27 Levine P, Kreuger G, Straus S. A postviral chronic fatigue syndrome: a round table. $\mathcal{F}$ Infect Dis 1989;160:722-4.

28 Ray C. Chronic fatigue syndrome and depression: conceptual and methodological ambiguities. Psychol Med 1991; 21:1-9.

29 Imboden JB, Canter A, Cluff LE. Convalescence from influenza: a study of the psychological and clinical determinants. Arch Intern Med 1961;108:115-21.

30 Stewart D. The changing face of somatisation. Somatics 1990;31:153-8. 